\title{
Uso de nuevo propante en fracturamiento hidráulico de pozos tight gas en el piedemonte colombiano
}

\author{
J. Portela ${ }^{1 *}$, J. Higuera ${ }^{1}$
}

doi: http://dx.doi.org/10.18273/revfue.v18n2-2020004 @) (1)

Forma de citar: Báez-Serrano, Portela, J., \& Higuera, J. (2020). Uso de nuevo propante en fracturamiento hidráulico de pozos tight gas en el piedemonte colombiano. Revista Fuentes, el reventón energético, 18(2), 57-67. https://doi.org/10.18273/revfue.v18n2-2020004

\section{Resumen}

El fracturamiento hidráulico de pozos es una técnica comprobada de mejora tanto de la productividad de hidrocarburos como de la inyectividad de agua y gas. El piedemonte llanero colombiano presenta una especial configuración de factores que dificultan tanto el proceso de inyección como el de producción, así como el proceso de fracturamiento hidráulico debido al alto tectonismo, la complejidad estructural, la baja permeabilidad y la profundidad de los yacimientos de interés.

Los pozos ubicados en el Piedemonte Colombiano y que fueron operados por BP, Equion y actualmente por Ecopetrol han sido fracturados en su gran mayoría para obtener tasas de producción económicamente rentables. Durante la historia de fracturamiento debido a la complejidad estructural, profundidad, los altos gradientes de fractura y las altas presiones de yacimiento; el propante utilizado fue del tipo sintético de mineral de bauxita cuyas propiedades físicas y químicas se ajustaban a los requerimientos técnicos de los pozos. Aunque este material presenta buenas propiedades de resistencia al esfuerzo de cierre, en general fueron requeridas altas concentraciones que, sumadas a la alta densidad del material, implicaron el uso de fluidos de acarreo altamente viscosos necesitando mayores concentraciones de polímero en el fluido y por ende mayores niveles de daño inducido a la formación tanto por fluidos como por sólidos generados debido al efecto de trituramiento ó 'crushing'. Durante los últimos años se ha detectado que las formaciones de Pauto y Floreña son muy sensibles a fluidos externos y debido a las bajas permeabilidades. En función de esto, el presente proyecto fue planeado para la aplicación de una nueva mezcla de fluidos de baja concentración polimérica y nuevos propantes de mejores propiedades de conductividad y densidad, que fueron probados a nivel de laboratorio previo a las aplicaciones en campo. Esta campaña de fracturamiento fue la primera en su tipo usando fluidos con las más bajas concentraciones en la historia de los campos y un referente en Latinoamérica generando excelentes resultados en términos financieros y de producción.

Palabras clave: pozos profundos, nuevo propante, pozos tight gas.

\section{Use of new propant in hydraulic fracturing of tight gas wells in colombian foothills}

\begin{abstract}
Hydraulic fracturing is a proven technique for improving both hydrocarbon productivity and water and gas injection. The Colombian foothills exhibit a special configuration of factors that increase the difficulty both during the injection and production processes, as well as in the hydraulic fracturing process due to high tectonism, structural complexity, low permeability and high depth of the reservoirs of interest.

The wells located in the Colombian foothills, operated in the past by BP \& Equion and currently by Ecopetrol, have been fractured in the majority to obtain economically profitable production rates. During the history of fracturing, structural complexity, high depths, high fracture gradients and high reservoir pressures have led to the usage of high strength / high density proppants such as bauxite whose physical and chemical properties complied with the technical requirements of the wells. Although this material has good properties of resistance upon closure stresses, high concentrations were normally required which, in addition to its high density, implied the use of highly viscous transport fluids requiring higher concentrations of polymer in the fluid and therefore higher levels of damage induced to formation by both, fluids and solids generated due to the 'crushing' effect of the proppant. In recent years, it has been documented that the Pauto and Floreña formations are very sensitive to external fluids due to their low permeabilities. Based on this, this project was planned for the application of
\end{abstract}

\footnotetext{
${ }^{1}$ Ecopetrol S.A. (*) E-mail:* fernando.portela33@outlook.com
} 
a new mixture of fluids with low polymer concentration and new proppants with better conductivity and lower density, which were tested at the laboratory level prior to field applications. This fracturing campaign was the first of its kind using fluids with the lowest concentrations in the history of the fields and is considered a benchmark for Latin America, due to its excellent results financially and production wise.

Keywords: tight gas fracturing, new proppant, deep wells.

\section{Introducción}

Los yacimientos apretados de gas o 'Tight Gas', requieren para su explotación del uso de técnicas que maximicen el área efectiva de flujo dado que sus bajas porosidades y permeabilidades imponen limitantes naturales para el flujo. De la ecuación de Darcy para flujo radial estable de gas, por ejemplo, puede inferirse que el pozo debe tener un área de flujo y presión altas para alcanzar caudales representativos. Dentro de las técnicas más usadas para lograr este objetivo, se encuentra el fracturamiento hidráulico y la perforación horizontal con o sin fractura hidráulica, y en general, el uso de una o varias de estas técnicas promoverá esquemas de producción económicamente rentables. En particular, los yacimientos naturalmente fracturados pueden beneficiarse de pozos horizontales de alta extensión o pozos multilaterales que promuevan la intersección de patrones de fracturas, sin embargo, el fracturamiento hidráulico casi siempre es necesario para maximizar su rentabilidad.

Las formaciones Tight Gas cuyo concepto fue desarrollado por Masters en 1979 pueden representarse mediante el "Triángulo de recursos" indicado en la figura 1 .

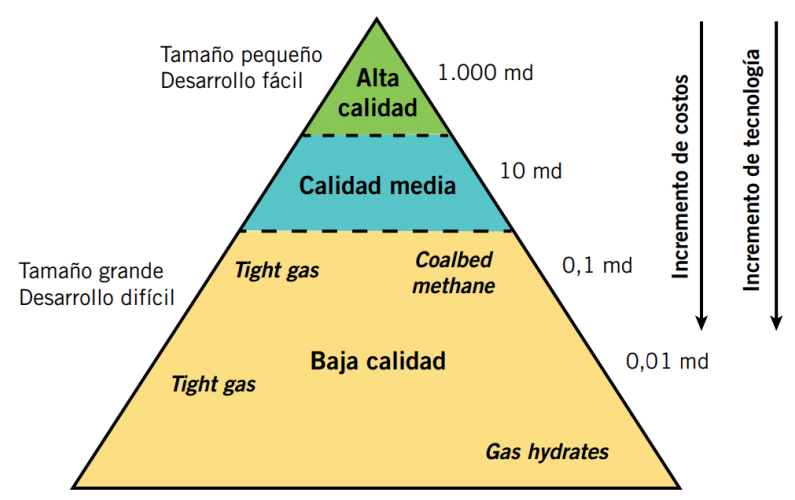

Figura 1. Triangulo de recursos para Gas Natural

Esta figura nos ayuda a estimar la inversión que se debe hacer para llegar a producir un yacimiento basado en su método de extracción y en su volumen. Puede concluirse así mismo que cada vez que uno se aleje del ángulo superior, las propiedades del yacimiento empeoran aumentando el tiempo al igual que la inversión que se necesita para producir el yacimiento. En resumen, se puede decir que los yacimientos de baja permeabilidad necesitan mejores tecnologías y mayores precios del barril para ser económicamente rentables.

Entre las consideraciones que se deben tener en cuenta en el momento del diseño de una fractura hidráulica en yacimientos tipos Tight Gas se mencionan las siguientes:

Diseño. Crear la mayor área de flujo posible es el objetivo principal de una fractura hidráulica en este tipo de yacimientos. En el caso yacimientos de gas condensado, la fractura debe ser lo suficientemente conductiva para movilizar las fases gas y aceite y generar suficiente área de contacto entre la fractura y el yacimiento (Archila, et al., 2014), (Ortiz, et al, 2010).

Fluidos de fractura. Dadas las propiedades geomecánicas de las rocas tipo 'tight gas', como el alto módulo de Young y nivel de anisotropía, la fractura creada tiende a ser larga y de poco espesor, lo cual genera una mayor cobertura areal pero exige una mejor conductividad en el cuerpo de la fractura. En función de esto, el fluido usado para el acarreo del material apuntalante debe ser limpio, minimizando los residuos de gel en la roca y en el cuerpo de la fractura, lo que exige utilizar sistemas de baja carga polimérica. (Carrascal, F. A., \& Contreras, Z. P., 2014), (Carrascal, F. A., et al., 2014).

Geomecánica. El diseño de una apropiada geometría de fractura requiere de una buena estimación de los esfuerzos in situ y de propiedades como el módulo de Young y la relación de Poisson con el fin de generar programas de bombeo que maximicen la cobertura de la fractura en espesores netos grandes (crecimiento vertical).

Propante. En sistemas tipo 'Tight Gas', debe promoverse una amplia cobertura areal más que una alta conductividad de la fractura (convencionalmente promovida mediante la técnica de 'Tip Screen Out' ó de 
empaquetamiento forzado), excepto en la zona cercana a la cara del pozo donde una buena conductividad puede minimizar efectos de turbulencia en ambientes de altas tasas de gas. En función de esto, el propante usado debe tener alta resistencia y promover conductividades suficientes aun a bajas concentraciones; en general, materiales de tipo sintético, que soporten los esfuerzos de cierre y los procesos cíclicos de apertura y cierre del pozo, son altamente recomendables hacia mayores tiempos de vida útil del pozo. (Ballesteros, R. A. M., \& González, F. E. C., 2016), (Carreño, et al., 2016), (Serrano, D. S. et al., 2013).
Área de Estudio. Los campos del Piedemonte Floreña y Complejo Pauto (figura 2), se encuentran ubicados en los llanos orientales de Colombia, 20 $\mathrm{Km}$ al noroccidente del municipio de Yopal, en el departamento del Casanare. Los pozos de estos campos se caracterizan por tener permeabilidades matriciales bajas $(\sim 1 \mathrm{mD})$ en función de lo cual el fracturamiento hidráulico es la técnica de estimulación que ha probado ser más efectiva para incrementar la producción de los pozos al crear un canal altamente conductivo que además permite contactar fracturas naturales existentes, ayudando a mejorar el flujo de fluidos del yacimiento al pozo y sobrepasando el daño de formación ocasionado durante la perforación. (Bustos, M., 2018).

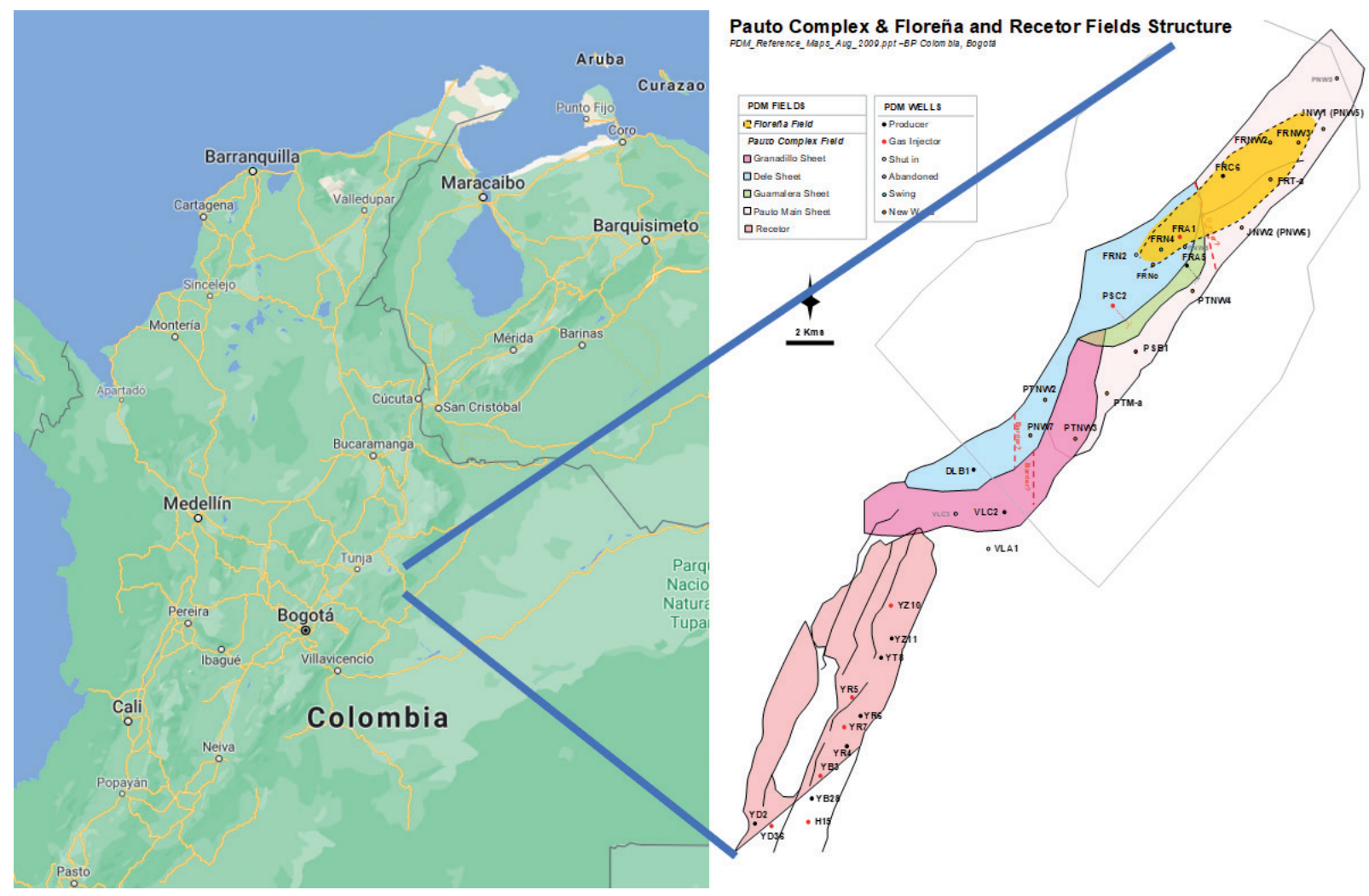

Figura 2. Localización geográfica Campo Piedemonte.

En cuanto a los rasgos geológicos, los yacimientos se caracterizan por ser altamente complejos estructuralmente, lo que induce alta anisotropía de esfuerzos dando lugar a amplias zonas de fracturas naturales y micro-fracturas en las zonas más profundas. La figura 2 ilustra un corte tipo de los reservorios, indicando la existencia de múltiples horizontes, presencia de fallas y pozos con altas desviaciones. 


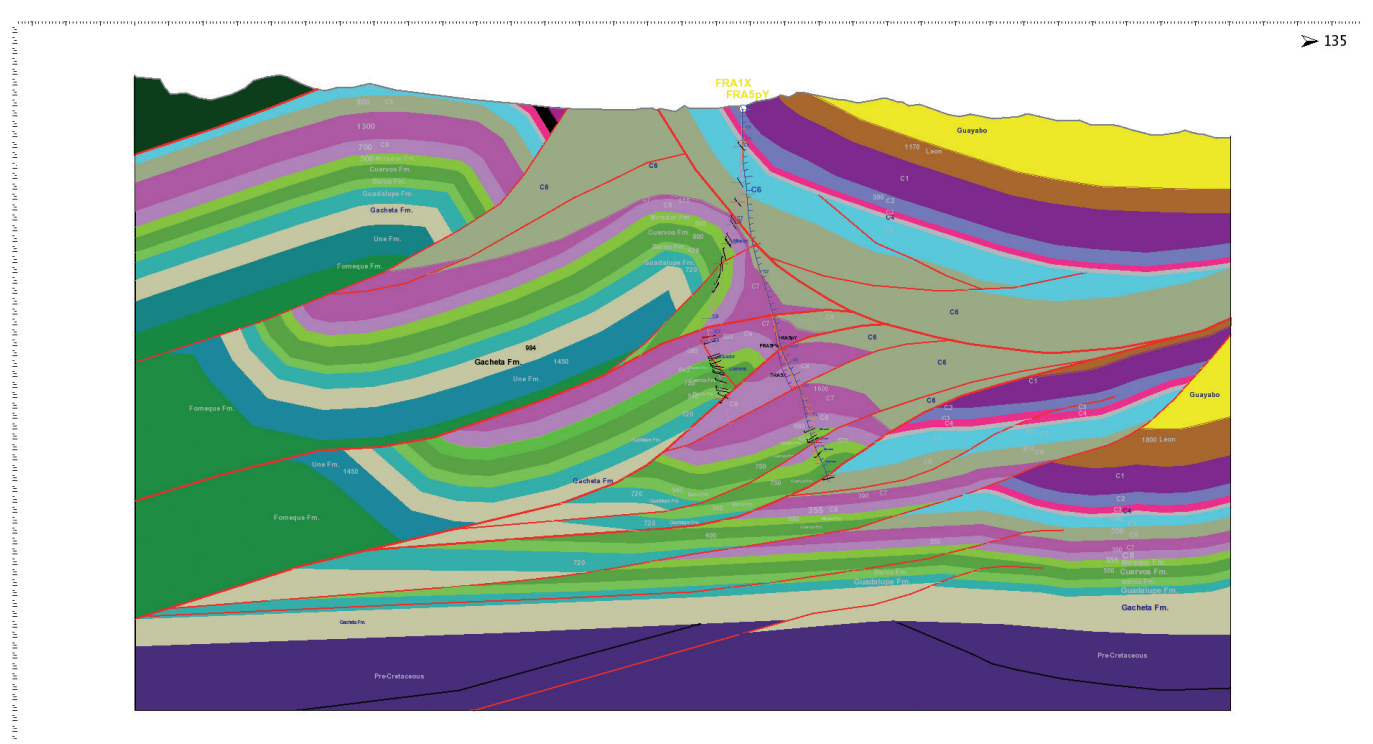

Figura 3. Columna estratigráfica Campo Piedemonte.

El estado mecánico de la figura 4 muestra un completamiento tipo, el cual indica altas profundidades $\mathrm{y}$ presencia de varias formaciones atravesadas por un mismo pozo. En superficie, se usan regularmente cabezales de $51 / 8$ " con rating de presión de 10,000 psi que en condiciones normales requerirá de un sistema

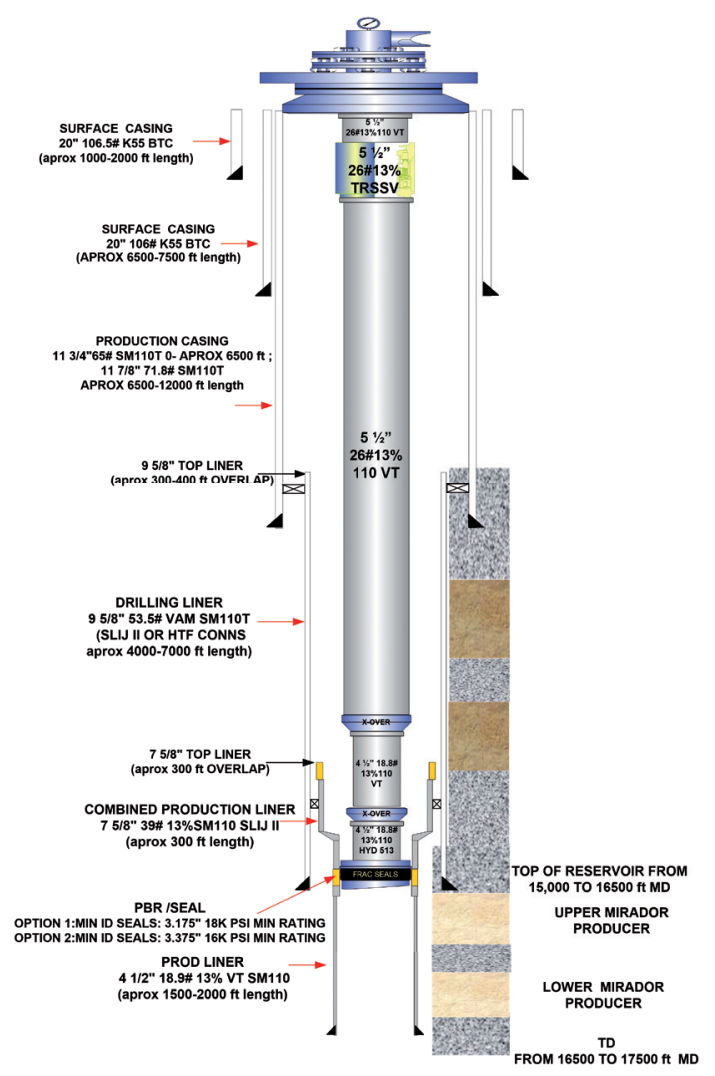

Figura 4. Estado mecánico genérico de los pozos. de protección tipo tree-saver o protector de árbol, debido a las altas presiones de bombeo esperadas durante el fracturamiento (hasta $25000 \mathrm{psi}$ ). La figura 5 , muestra un set de propiedades geomecánicas tipo en la zona objetivo.
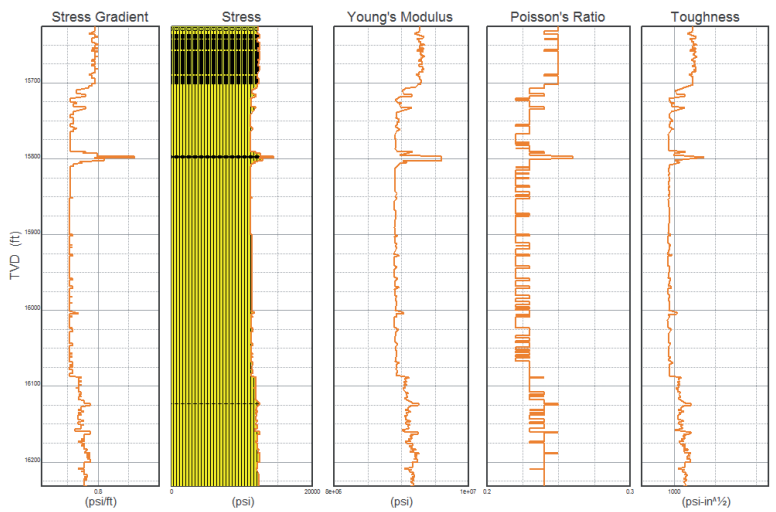

Figura 5. Propiedades de Roca

Desde el inicio de producción y hasta la fecha, los campos del Piedemonte reportan 184 trabajos de fracturamiento hidráulico. Las condiciones petrofísicas, la temperatura de los yacimientos productores, la profundidad y los esfuerzos in-situ, implicaron el uso de propantes basados en mineral de Bauxita, de buenas propiedades de conductividad y resistencia, pero de alta gravedad especifica (3.65), demandando un fluido de acarreo altamente viscoso y de alta carga polimérica. Lo anterior, implicó mayores costos operativos relacionados con largos tiempos para la operación de limpieza del material propante y la fase 
de flowback de la fractura. En algunas operaciones, fueron también documentados eventos asociados con daño remanente por polímero y bloqueo líquido, este último debido a efectos capilares en rocas de baja permeabilidad y porosidad (ver tabla 1).

De otro lado, la geometría de los pozos y el alto nivel de anisotropía de esfuerzos, generaron retos adicionales, implicando en algunos casos, altos gradientes de fractura y de cierre, como se indica en la figura 6.
Tabla 1. Propiedades Petrofísicas Campo Piedemonte.

\begin{tabular}{cccc}
\hline $\begin{array}{c}\text { Pozo } \\
\text { Forma }\end{array}$ & $\begin{array}{c}\text { Perm } \\
\text { Matricial } \\
\text { K md }\end{array}$ & $\begin{array}{c}\text { Porosidad } \\
\text { \% }\end{array}$ & $\begin{array}{c}\text { Presión } \\
\text { Yacimiento } \\
\text { psi }\end{array}$ \\
\hline 1A & 1,5 & 3,5 & 6150 \\
1B & 1,5 & 3,5 & 6150 \\
2 & 1 & 3 & 5100 \\
3 & 0,5 & 3 & 4800 \\
1C & 0,8 & 3,2 & 4850 \\
\hline
\end{tabular}

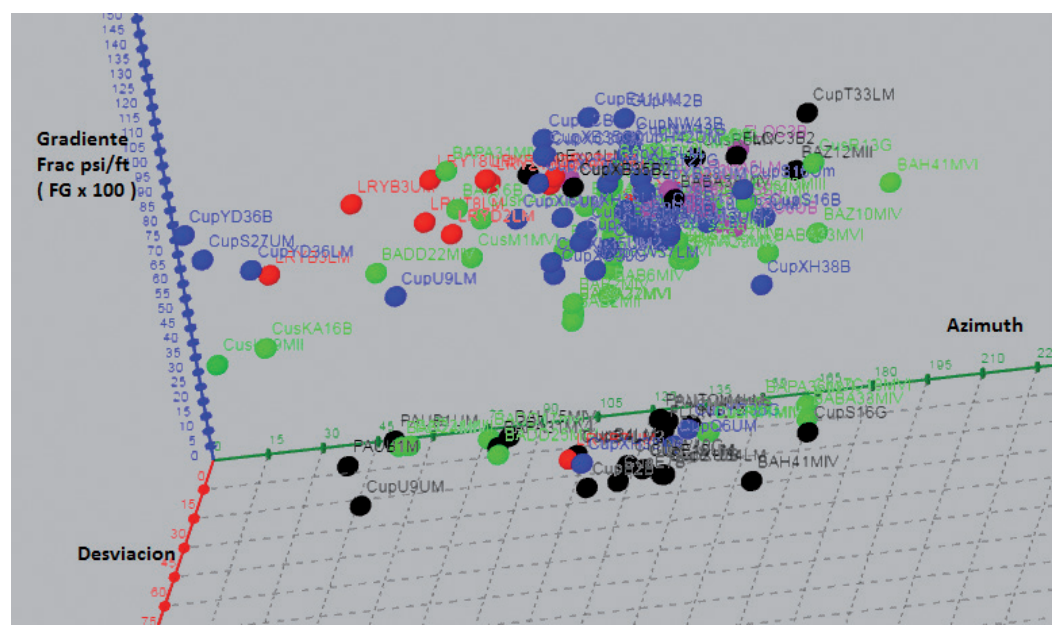

Figura 6. Gradiente de Fractura x 100 Vs Azimuth Vs Desviación.

\section{Antecedentes}

Todos los pozos fueron perforados y cañoneados promoviendo condiciones óptimas para el fracturamiento hidráulico desde su inicio, es decir, buscando bajas desviaciones o alineación del pozo con la dirección del esfuerzo máximo en la zona objetivo.
Las profundidades típicas de cañoneo oscilaron entre 16.000 y $18.000 \mathrm{fts}$ MD (14.000 - $16.000 \mathrm{fts}$ TVD). Como fue ya descrito, el propante usado durante el $98 \%$ de los fracturamientos hidráulicos realizados previos a este estudio fue del tipo mineral de Bauxita cuyas propiedades se indican en la figura 7 .
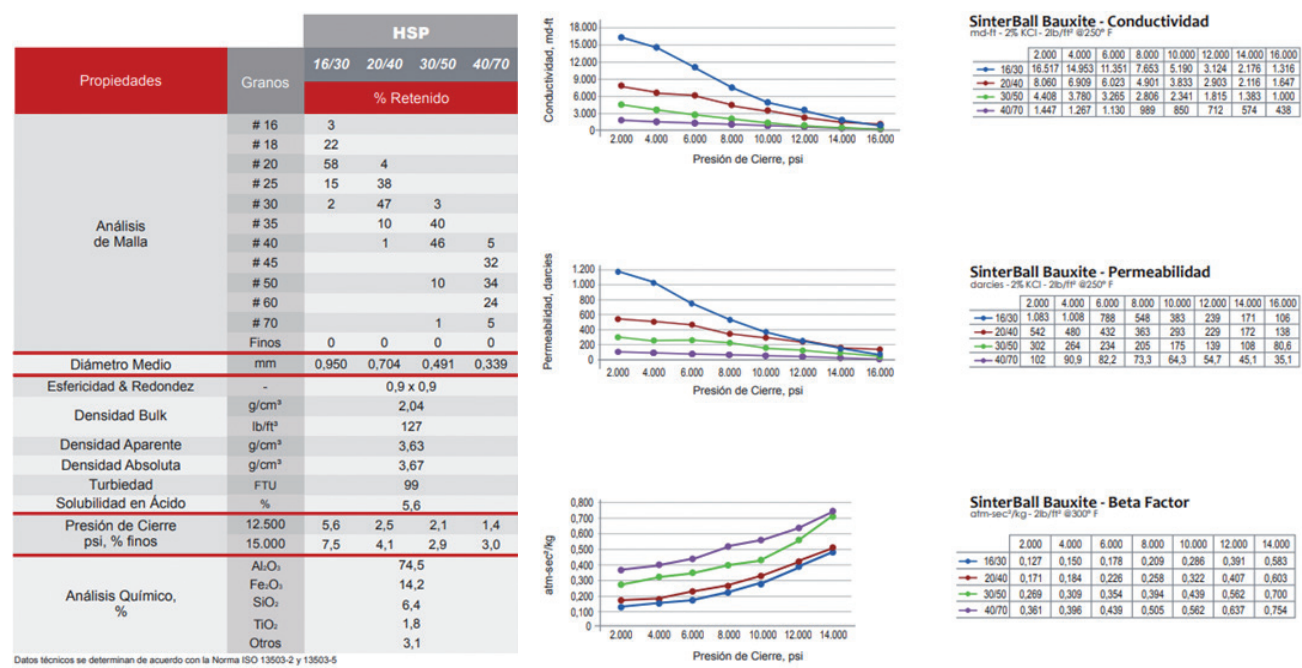

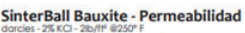

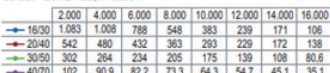

SinterBall Bauxite - Beta Factor

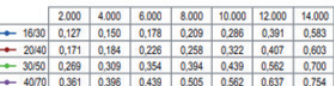

Figura 7. Propiedades Fisicoquímicas de propante de alta resistencia a base de mineral de bauxita 
El propante usado, corresponde a tamaños de malla entre $16 / 30$ y 20/40, con densidad aparente de 3.63 requiriendo el uso de fluidos de alta carga polimérica (típicamente 45 libras por cada mil galones de lechada ó 45 ppt) para alcanzar la viscosidad necesaria para su acarreo a las concentraciones de propante normalmente requeridas de hasta $8 \mathrm{ppg}$. El perfil reológico de un fluido tipo se muestra en la Figura 8 y el nivel de daño inducido por el gel de fractura se muestra en la figura 9, condiciones que sumadas a las propiedades del propante tipo bauxita, son tomadas como base para el presente estudio de optimización del sistema de fracturamiento que se describe a continuación.

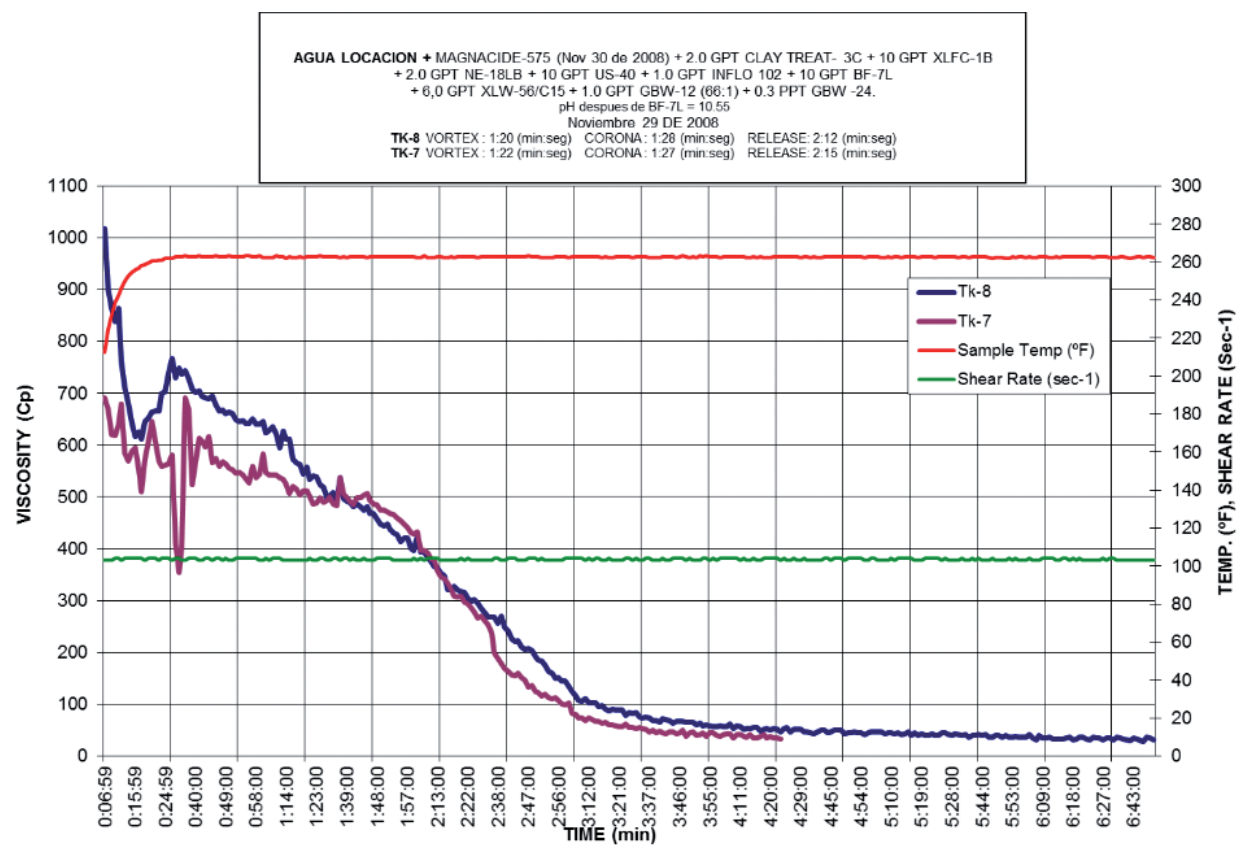

Figura 8. Propiedades Reológicas Fluido de 40 libras x mil galones

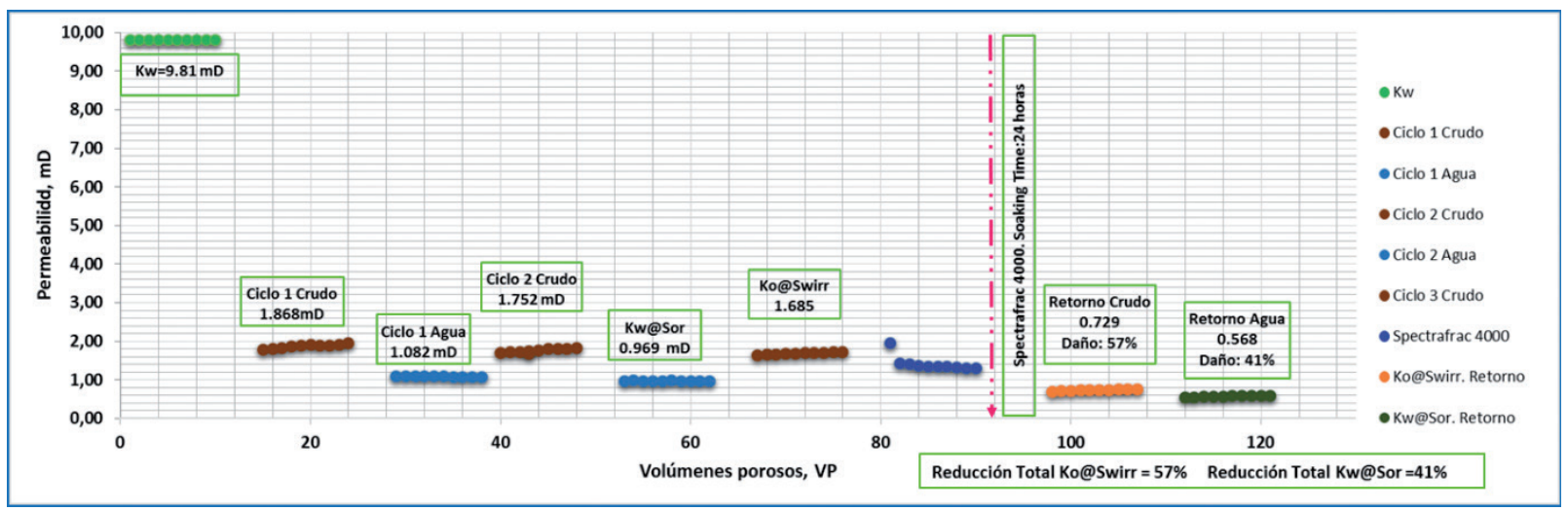

Figura 9. Desplazamiento de Fluido de 40 ppt en rocas representativas.

NOTA: Según las figuras 8 y 9 y para el nuevo sistema, serían requeridas reologías estables por encima de $500 \mathrm{cp}$ en 1 hora a esfuerzos de corte de 100 sec-1 (en Reómetro tipo Fann 50) así como daños menores al 57\% post invasión de fluido de fractura.

\section{Diseño de la Solución}

A continuación, se presentan los cambios efectuados tanto sobre el fluido de acarreo como sobre el propante hacia la minimización de daño inducido y la optimización del programa de bombeo, siempre manteniendo el objetivo de tener una adecuada geometría de fractura con suficiente conductividad. 


\section{Fluidos}

Las condiciones objetivo para el fluido fueron mantener una reología estable de al menos $500 \mathrm{cp}$ de viscosidad después de 1 hora a esfuerzos de corte de $100 \mathrm{sec}-1$ (mediciones hechas en Reómetro tipo Fann 50, figura 8). A partir de este tiempo, el fluido debería iniciar su proceso de rompimiento hasta regresar a condiciones de gel lineal (pérdida de condiciones de entrecruzado) para facilitar el proceso de producción después del tratamiento (flowback). Todo lo anterior, fue probado teniendo en cuenta un gradiente Geotérmico de 1.12 ${ }^{\circ} \mathrm{F} / 100 \mathrm{ft}$, que, para el área de estudio, representa una temperatura estática de fondo de $260{ }^{\circ} \mathrm{F}$. Adicionalmente, el fluido utilizado debía generar el menor impacto posible sobre la permeabilidad para lo cual se corrieron ensayos tipo coreflood con diferentes formulaciones hasta encontrar aquella de mejor desempeño. El impacto en permeabilidad de los fluidos base, es decir, no optimizados, se muestra en la figura 9, indicando daños de aproximadamente $57 \%$ en la permeabilidad efectiva al petróleo post-inyección del fluido de fractura. El desempeño del fluido optimizado, de menor carga polimérica, se ilustra en las figuras 10 y 11, notándose en el primer caso, un desempeño reológico adecuado (viscosidad mayor a $500 \mathrm{cp} \mathrm{@} 1$ hr) pero a cargas poliméricas más bajas, de entre 25 y 30 libras por cada 1000 galones de lechada (25$30 \mathrm{ppt}$ ); y en el segundo caso, reportando niveles de daño mucho menores, de $24 \%$ (vs $57 \%$ base). También se muestra en la figura 11, como la inclusión de un sistema oxidante para el rompimiento de polímero residual, logra un daño residual de apenas un $9 \%$.
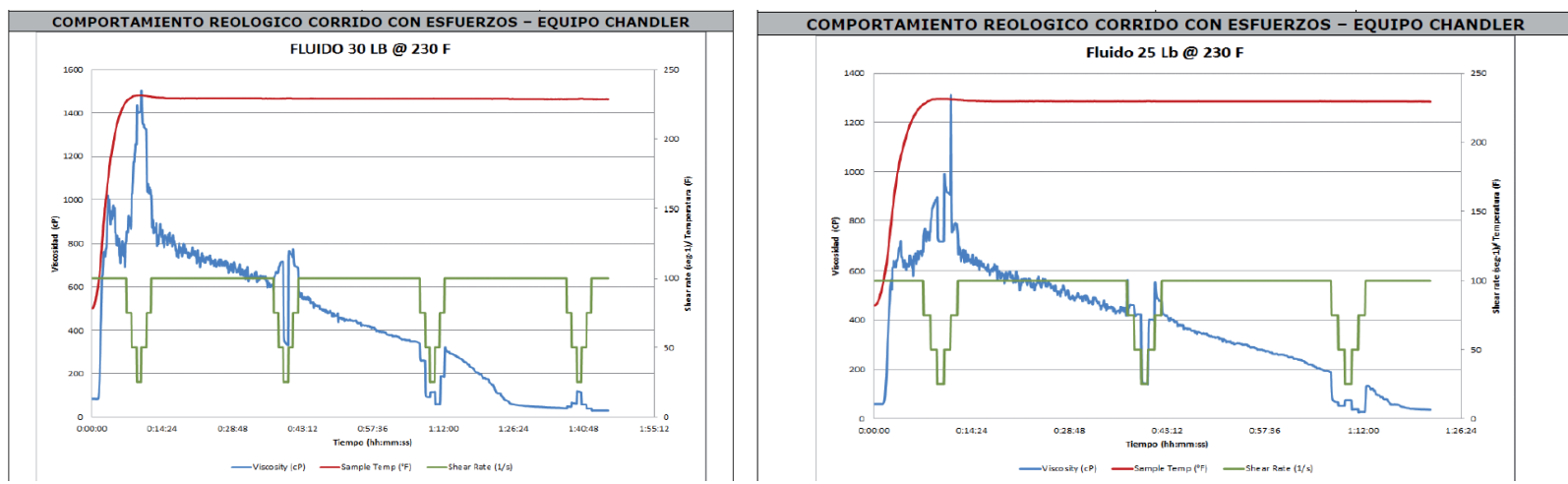

Figura 10. Propiedades Reológicas Fluido de 25 y 30 libras x mil galones

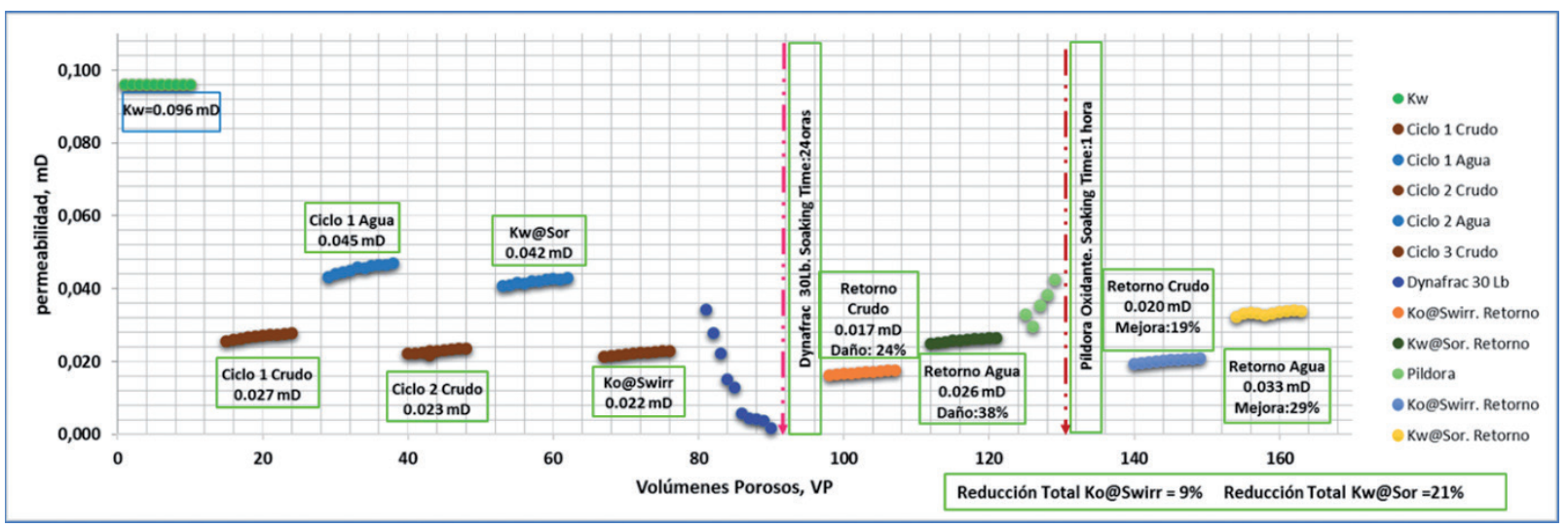

Figura 11. Desplazamiento de Fluido optimizado de 30 libras de polímero en rocas representativas. Daño del $24 \%$ en Ko post inyección de gel. Daño final del 9\% en Ko post-inyección de píldora oxidante para rompimiento del polímero residual.

\section{Propante}

Para las propiedades geomecánicas indicadas en la figura 5 y las características $\mathrm{p}$ etrofísicas de la roca (Tabla 1), el propante HSP a base de mineral de Bauxita cumplió con los requerimientos técnicos de alta resistencia y conductividad como se ve en la Figura 7. Este propante, combinado con el fluido base (figuras 8 y 9) se usaron en la gran mayoría de tratamientos previos a este estudio a partir del cual, fueron evaluados nuevos desarrollos tecnológicos ofrecidos por las compañías fabricantes de propante. 
Luego de una exhaustiva investigación de mercado, se seleccionó un nuevo material que denominaremos LD25 cuyas propiedades se indican en la figura 12.

Long-term conductivity

\begin{tabular}{|c|c|c|}
\hline Closure stress & Reference conductivity*, md-ft & \\
\hline [psi] & 25 Mesh & 35 Mesh \\
\hline 2,000 & 9,000 & 4,100 \\
\hline 4,000 & 7,600 & 3,600 \\
\hline 6,000 & 6,100 & 3,150 \\
\hline 8,000 & 4,400 & 2,600 \\
\hline 10,000 & 3,000 & 2,000 \\
\hline 12,000 & 1,900 & 1,400 \\
\hline 14,000 & 1,150 & 900 \\
\hline \multicolumn{3}{|c|}{$\begin{array}{l}\text { Reference conductivity and permeability are measured with a single phase fluid under laminar } \\
\text { flow conditions in accordance with IIO } 13503-5 \text {. In an actual fracture, the effective conductivity will } \\
\text { be much lower due to non-Darcy and multiphase flow effects. For more information, please refer } \\
\text { to SPE Paper } \$ 106301 \text {. }\end{array}$} \\
\hline Closure stress & Reference permeability, Darcies & \\
\hline [psi] & 25 Mesh & 35 Mesh \\
\hline 2,000 & 495 & 240 \\
\hline 4,000 & 425 & 213 \\
\hline 6,000 & 345 & 187 \\
\hline 8,000 & 255 & 155 \\
\hline 10,000 & 175 & 120 \\
\hline 12,000 & 115 & 88 \\
\hline 14,000 & 70 & 60 \\
\hline
\end{tabular}

\section{Physical and chemical properties}

Typical sieve analysis [weight $\%$ retained]

$\frac{\frac{\text { U.S. Mesh [mesh] }}{-20+25 \text { mesh }}}{\frac{-30+35 \text { mesh }}{-850+710}} \frac{\frac{\text { Microns }}{-600+500}}{\frac{-8525}{100}} \frac{0}{810}$

\section{API/ISO crush test}

\% by weight fines generated 12,500 psi 8

Sizing requirements:

These specifications meet the recommended practices as detailed in ISO $13503-2$.

Typical additional properties

\begin{tabular}{ll}
\hline Roundness & $\frac{0.9}{0.9}$ \\
\hline Sphericity & $\frac{104}{1.6}$ \\
\hline Bulk density $[\mathrm{lb} / \mathrm{ft}]$ & $\frac{1.67}{[\mathrm{~g} / \mathrm{cm}]}$ \\
\hline Chemistry & $>50 \%$ \\
& Alumina \\
\hline
\end{tabular}

$\frac{\text { Apparent specific gravity }}{\text { Absolute volume [gal//b] }}$
$\begin{aligned} & \text { Solubility in } 12 / 3 \mathrm{HCV} / \mathrm{HF} \\ & \text { acid [\% weight loss] }\end{aligned}$

Figura 12. Propiedades Fisicoquímicas de propante de nueva tecnología (LD 25).

De esta figura se nota que la gravedad especifica de este nuevo propante es de 2.83 en comparación a 3.67 del propante anterior (un $23 \%$ menos de peso) con propiedades comparables o incluso superiores de resistencia y conductividad, como se muestra en la figura 13, y de menores efectos de turbulencia, indicados en un menor factor beta ilustrado en la figura 14; todo esto, a las condiciones de esfuerzo efectivo típicas que se muestran en la tabla 2. De un lado, nótese que según la tabla 2, la presión efectiva en fondo oscila entre los valores de presión efectiva 1 y presión efectiva 2 (5800-9400 psi), representados en el recuadro azul de la figura 14, indicando valores de conductividad para el propante LD25 superiores a los de su predecesor, HSP base mineral de bauxita. De otro lado, el propante LD25, al tener un solo tamaño (mono malla) y una mejor esfericidad (figura 15), genera un menor factor beta (o de turbulencia), clave en pozos de alto flujo de gas objeto del presente estudio. Finalmente, y complementando las ventajas mencionadas, se prevé que un menor peso del propante, maximiza los efectos convectivos en el cuerpo de la fractura, optimizando el transporte y cobertura del material de sostén. Todo lo anterior, permitió usar un fluido de acarreo con menos carga polimérica y de menor daño, ya ilustrado en las figuras 10 y 11.

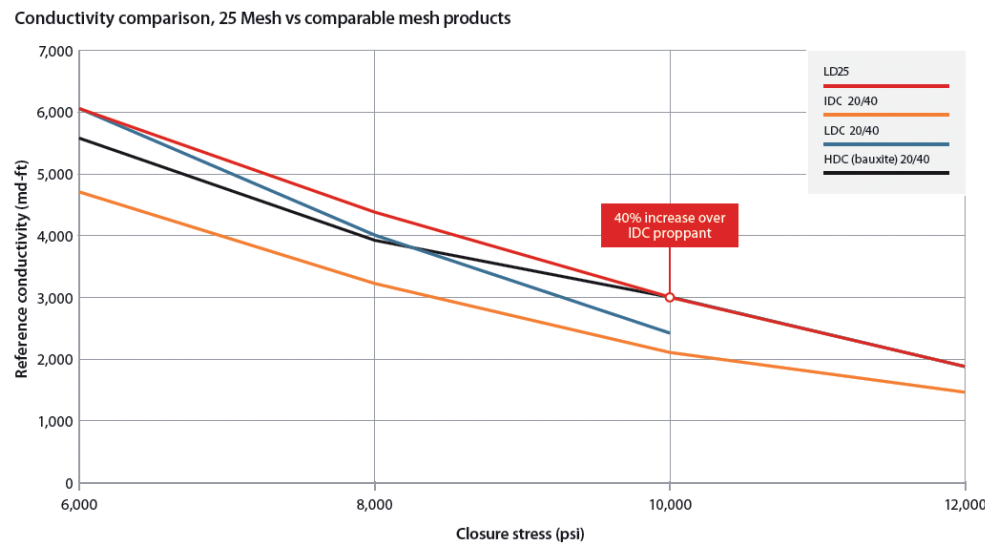

Figura 13. Comparación HSP (High Strenght Proppant) Vs Nuevo propante malla 25 (LD25). 
Tabla 2. Presión efectiva en fondo a condiciones estáticas y dinámicas de los pozos.

\begin{tabular}{cccccccc}
\hline $\begin{array}{c}\text { Pozo } \\
\text { Forma }\end{array}$ & $\begin{array}{c}\text { Perm Matricial } \\
\text { K md }\end{array}$ & $\begin{array}{c}\text { Porosidad } \\
\text { \% }\end{array}$ & $\begin{array}{c}\text { Presión } \\
\text { Yacimiento } \\
\text { psi }\end{array}$ & $\begin{array}{c}\text { Presion fondo } \\
\text { fluyendo psi }\end{array}$ & $\begin{array}{c}\text { Grafiente } \\
\text { Frac Psi/ft }\end{array}$ & $\begin{array}{c}\text { Presión } \\
\text { efectiva 1 psi }\end{array}$ & $\begin{array}{c}\text { Presión efectiva } \\
\text { 2 psi }\end{array}$ \\
\hline 1A & 1,5 & 3,5 & 6150 & 2800 & 0,75 & 5850 & 9200 \\
1B & 1,5 & 3,5 & 6150 & 2800 & 0,75 & 5850 & 9200 \\
2 & 1 & 3 & 5100 & 2600 & 0,75 & 6900 & 9400 \\
3 & 0,5 & 3 & 4800 & 3000 & 0,75 & 7200 & 9000 \\
1C & 0,8 & 3,2 & 4850 & 2800 & 0,75 & 7150 & 9200 \\
\hline
\end{tabular}
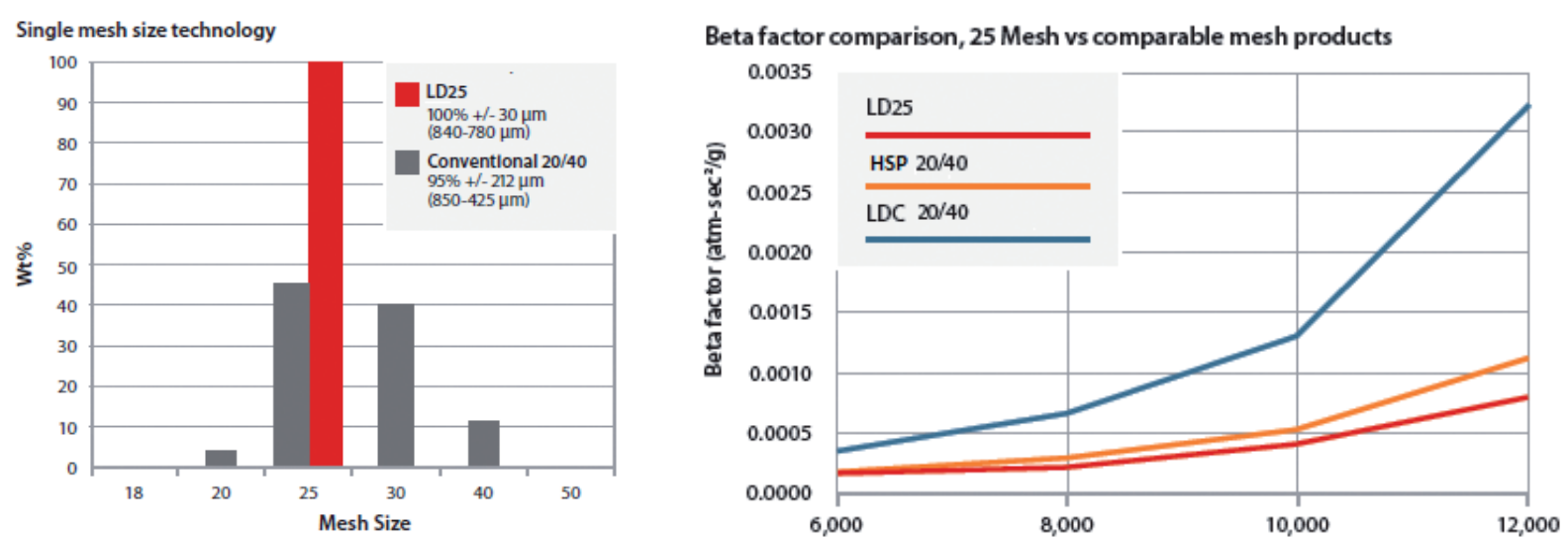

Figura 14. Comparación HSP (High Strenght Proppant) Vs Nuevo propante malla 25 (LD25).

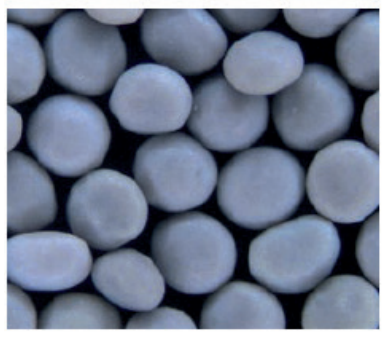

LD25
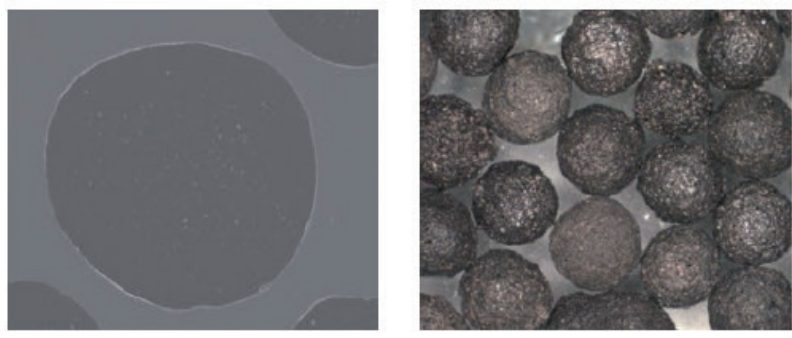

Standard proppant

Figura 15. Foto granular. Comparación HSP (High Strenght Prppant) Vs Nuevo propante malla 25 (LD25).

\section{Pruebas de Campo}

Después de la optimización tanto del fluido como del material propante y según lo anteriormente descrito, se realizaron 5 trabajos de fracturamiento hidráulico resumidos en la tabla 3 cuyo desempeño se describe en la sección de resultados.

Tabla 3. Resumen Operativo de la Nueva Campaña de Fracturamiento Hidráulico.

\begin{tabular}{crccc}
\hline $\begin{array}{c}\text { Pozo } \\
\text { Forma }\end{array}$ & $\begin{array}{c}\text { Libras } \\
\text { propante lbs }\end{array}$ & $\begin{array}{c}\text { Carga Polimétrica } \\
\text { ppt }\end{array}$ & $\begin{array}{c}\text { Esfuezo de cierre } \\
\text { psi7ft }\end{array}$ & $\begin{array}{c}\text { Concentración en cara } \\
\text { de fm ppa }\end{array}$ \\
\hline 1A & 40.000 & $30-25$ & 0,59 & 3 \\
1B & 133.200 & $35-30$ & 0,56 & 6 \\
2 & 87.000 & 32 & 0,81 & 6 \\
3 & 7.000 & 30 & 0,7 & 1,5 \\
1C & 32.600 & 20 & 0,76 & 3 \\
\hline
\end{tabular}




\section{Resultados}

La tabla 4 resume los resultados obtenidos durante la nueva campaña de fracturamiento con el sistema fluidopropante optimizado cuyos principales elementos se describen a continuación:

- Un total de 299,800 lbs de LD25 fueron colocadas en formación en 5 trabajos y 3 pozos en un lapso de 2 años a partir del proceso de cambio de propante y fluido. No se presentaron incidentes de HSE durante la ejecución de los trabajos.

- El análisis económico de la nueva campaña de fracturamiento, indicó NPVs positivos en todos los casos cumpliendo con los lineamientos de
Disciplina de Capital del operador.

- La baja densidad del propante usado, facilitó el levantamiento de la arena remanente en la tubería después del trabajo principal, reduciendo los tiempos de intervención de 4 - 6 días (caso base - propante HSP) a entre 1 y 2 días (propante LD25), generando así, ahorros en el costo total de operación y menores impactos de producción por cierre del pozo.

- El beneficio total de producción incremental promedio fue de 4790 bopd asociado a la nueva campaña de fracturamiento, con un total de 299,800 lbs de LD 25 en formación.

Tabla 4. Resumen Operativo y de Producción.

\begin{tabular}{cccccccc}
\hline $\begin{array}{c}\text { Pozo } \\
\text { Forma }\end{array}$ & $\begin{array}{c}\text { Libras } \\
\text { propante } \\
\text { lbs }\end{array}$ & $\begin{array}{c}\text { Carga } \\
\text { Polimétrica } \\
\text { ppt }\end{array}$ & $\begin{array}{c}\text { Esfuezo de } \\
\text { cierre } \\
\text { psifft }\end{array}$ & $\begin{array}{c}\text { Concentración en } \\
\text { cara de fm ppa }\end{array}$ & $\begin{array}{c}\text { Prod Crudo } \\
\text { Pre-Frac } \\
\text { bopd }\end{array}$ & $\begin{array}{c}\text { Prod Crudo } \\
\text { Post-Frac } \\
\text { bopd }\end{array}$ & Beneficio \\
\hline 1A & 40.000 & $30-25$ & 0,59 & 3 & 0 & 1800 & 1800 \\
1B & 133.200 & $35-30$ & 0,56 & 6 & 500 & 1800 & 1300 \\
2 & 87.000 & 32 & 0,81 & 6 & 100 & 450 & 350 \\
3 & 7.000 & 30 & 0,7 & 1,5 & 0 & 1100 & 1100 \\
1C & 32.600 & 20 & 0,76 & 3 & 0 & 240 & 240 \\
\hline
\end{tabular}

\section{Conclusiones}

- A través de un adecuado aseguramiento experimental, se diseñó un nuevo fluido de fractura con cargas poliméricas de entre 25 y 30 libras por cada mil galones (ppt) con menor daño de formación asociado.

- Un nuevo propante, de menor densidad, mejor esfericidad y selección (mono-malla), denominado LD25, probó su éxito operativo abriendo nuevas posibilidades para la viabilidad de campañas de fracturamiento en Colombia y Suramérica.

- Se obtuvo un beneficio neto promedio de 4790 bopd después de la campaña de fracturamiento y se redujeron los tiempos operativos asociados con la fase de limpieza del pozo por el uso de un propante más liviano.

- El uso de este propante LD25 en Colombia, abre nuevas posibilidades para su implementación en Suramérica y en el país, en diferentes activos de la compañía.

- Esta nueva tecnología apalancará el desarrollo de nuevos pozos en el área bajo la técnica de estimulación hidráulica y permitirá el desarrollo de más activos para la compañía maximizando los caudales de producción.

\section{Nomenclatura}

bopd=barriles de aceite por día

psi=Libras por pulgada cuadrada. Sistema Ingles

$\mathrm{ft}=$ Pies. Sistema Ingles

$\mathrm{ppt}=$ Libras por cada mil galones. Sistema Ingles

ppa $=$ Libras por galón adherido de propante. Sistema Ingles.

lbs $=$ libras. Sistema Ingles

gls $=$ Galones. Sistema Ingles

bbls=barriles. Sistema Ingles

\section{Referencias bibliográficas}

Archila, J. L., Romero, N. A., Calvete, F. E., \& Ardila, S. A. (2014). ESTUDIO TÉCNICO-FINANCIERO PARA LA VIABILIDAD DE UN TRATAMIENTO DE FRACTURAMIENTO HIDRÁULICO EN CAMPO ESCUELA COLORADO. Fuentes, el reventón energético, 12(1). 
Ballesteros, R. A. M., \& González, F. E. C. (2016). Modelado del efecto de la movilidad del banco de finos de propante sobre las tendencias de producción en pozos hidráulicamente fracturados. Fuentes, el reventón energético, 14(2), 41-49.

Bustos, M. (2018). Selección de pozos candidatos a fracturamiento hidráulico en el campo Gustavo Galindo Velasco. Fuentes, el reventón energético, 16(1), 81-86.

Carrascal, F. A., \& Contreras, Z. P. (2014). Química aplicada al control de calidad de fluidos: Éxito del fracturamiento hidráulico. Fuentes, el reventón energético, 12(2).

Carrascal, F. A., Contreras, Z. D. P. P., \& Velasco, D. M. (2014). Evaluación de la eficiencia de rompedores de fluidos de fractura base agua. Fuentes: El reventón energético, 12(1), 4.

Carreño, L. D. G., Duarte, J. B. D., Hernández, E. A. G., Tarazona, D. M., \& Perez, E. R. (2016). Evaluación de alternativas de costo para el suministro de propantes a un yacimiento no convencional en Colombia. Fuentes: El reventón energético, 14(1), 53-66

Jose Fernando Portela. TEC 369. Nuevas formulaciones Fluidos de control Piedemonte. 2017.

Jose Fernando Portela. TEC 358. Estimulación Hidráulica en un pozo inyector de baja permeabilidad en hueco abierto. 2017.

Kenneth G. Nolte. Reservoir Stimulation. John Wiley \& Sons. 2000.

Michael J. Economides. Modern Fracturing. ET Publishing. 2007.

Ortiz, M. M., Calvete, F., Calderón, Z., Saavedra, N. F., \& Corzo, R. (2010). Refracturamiento hidráulico:"una exitosa técnica de estimulación de pozos". Fuentes, el reventón energético, 8(2).

Serrano, D. S., Gómez, C. A. P., Rueda, R. C., \& Núñez, R. D. C. (2013). Implementación de propantes ultralivianos en el fracturamiento hidráulico de pozos. Fuentes: El reventón energético, 11(1), 1.

Fecha de recepción: 26 de Noviembre de 2019

Fecha de aceptación: 18 de noviembre de 2020 